\title{
Gluon Shadowing in Heavy-Flavor Production at the LHC
}

\author{
Aleksander Kusina, ${ }^{1}$ Jean-Philippe Lansberg, ${ }^{2}$ Ingo Schienbein, ${ }^{3}$ and Hua-Sheng Shao ${ }^{4,5}$ \\ ${ }^{1}$ Institute of Nuclear Physics, Polish Academy of Sciences, ul. Radzikowskiego 152, 31-342 Cracow, Poland \\ ${ }^{2}$ IPNO, CNRS-IN2P3, Univ. Paris-Sud, Université Paris-Saclay, 91406 Orsay Cedex, France \\ ${ }^{3}$ Laboratoire de Physique Subatomique et de Cosmologie, Université Grenoble-Alpes, \\ CNRS/IN2P3, 53 avenue des Martyrs, 38026 Grenoble, France \\ ${ }^{4}$ Sorbonne Universités, UPMC Univ. Paris 06, UMR 7589, LPTHE, F-75005, Paris, France \\ ${ }^{5}$ CNRS, UMR 7589, LPTHE, F-75005, Paris, France
}

(Received 20 December 2017; revised manuscript received 26 April 2018; published 3 August 2018)

\begin{abstract}
We study the relevance of experimental data on heavy-flavor $\left[D^{0}, J / \psi, B \rightarrow J / \psi\right.$ and $\Upsilon(1 S)$ mesons $]$ production in proton-lead collisions at the LHC to improve our knowledge of the gluon-momentum distribution inside heavy nuclei. We observe that the nuclear effects encoded in both most recent global fits of nuclear parton densities at next-to-leading order (nCTEQ15 and EPPS16) provide a good overall description of the LHC data. We interpret this as a hint that these are the dominant ones. In turn, we perform a Bayesian-reweighting analysis for each particle data sample which shows that each of the existing heavyquark(onium) data set clearly points - with a minimal statistical significance of $7 \sigma$ - to a shadowed gluon distribution at small $x$ in the lead. Moreover, our analysis corroborates the existence of gluon antishadowing. Overall, the inclusion of such heavy-flavor data in a global fit would significantly reduce the uncertainty on the gluon density down to $x \simeq 7 \times 10^{-6}$ - where no other data exist—while keeping an agreement with the other data of the global fits. Our study accounts for the factorization-scale uncertainties which dominate for the charm(onium) sector.
\end{abstract}

DOI: 10.1103/PhysRevLett.121.052004

Introduction-Parton-distribution functions (PDFs), describing the longitudinal-momentum distributions of quarks and gluons inside hadrons, provide the essential link between the measurable hadronic cross sections and the perturbatively calculable cross sections of high-energy processes induced by quarks and gluons. The precise determination of PDFs of protons, $f_{i}^{p}$, is an extremely active area of research where several groups perform global analyses of a wide variety of experimental hard-process data. The modern global analyses [1-6] have evolved into impressive ventures with state-of-the-art perturbative calculations and sophisticated statistical methods to extract optimum PDFs along with their uncertainties.

The situation is more challenging-but not less interesting-for PDFs of nucleons inside nuclei, $f_{i}^{[p, n] / A}$, with nuclear data significantly more complex to collect and with two additional degrees of freedom, the number of protons $(Z)$ and neutrons $(N=A-Z)$ in the studied nuclei. Nuclear PDFs (nPDFs) are key ingredients to use perturbative probes of the quark-gluon plasma produced in

Published by the American Physical Society under the terms of the Creative Commons Attribution 4.0 International license. Further distribution of this work must maintain attribution to the author(s) and the published article's title, journal citation, and DOI. Funded by SCOAP ${ }^{3}$. ultrarelativistic nucleus-nucleus collisions at RHIC and the LHC [7]. As such, their determination goes even beyond the understanding of the nucleus content in terms of quarks and gluons. Since the early 1980s, we know that the nuclei are not a simple collection of free nucleons, and nPDFs are not equal to a sum of nucleon PDFs. In fact, the corresponding analyses rather bear on nuclear-modification factors (NMF), like in lepton-nucleus $(\ell A)$ collisions $R\left[F_{2}^{\ell A}\right]=F_{2}^{\ell A} /\left(Z F_{2}^{\ell p}+(A-Z) F_{2}^{\ell n}\right)$ for the deep-inelastic scattering (DIS) structure function $F_{2}$ and parton-level NMFs $R_{i}^{A}\left(x, \mu_{F}\right)=f_{i}^{A} /\left(Z f_{i}^{p}+N f_{i}^{n}\right)$ with $f_{i}^{A} \equiv Z f_{i}^{p / A}+$ $N f_{i}^{n / A}(i=g, q, \bar{q})$, instead of the absolute nPDFs.

Based on earlier studies of $F_{2}$ [8-14], one knows that, for the quarks, (i) $R_{q}^{A}>1$ for $x \gtrsim 0.8$ (Fermi-motion region), (ii) $R_{q}^{A}<1$ for $0.25 \lesssim x \lesssim 0.8$ (EMC region), (iii) $R_{q}^{A}>1$ for $0.1 \lesssim x \lesssim 0.25$ (antishadowing region), and (iv) $R_{q}^{A}<1$ for $x \lesssim 0.1$ (shadowing region) where different physics mechanisms were proposed to explain this behavior. At medium and large longitudinal-momentum fractions, $x, R_{q}^{A}$ is usually explained by nuclear-binding and medium effects and the Fermi motion of the nucleons [15] but a fully conclusive picture has not yet emerged after the discovery of the EMC effect [16]. At small $x$, coherent scatterings inside the nucleus explain the observed suppression of $F_{2}$, referred to as shadowing. Antishadowing is even less understood. Therefore, just like in the nucleon case, 
nPDFs are determined by performing global analyses of experimental data [17-21].

Compared to the quark content—directly probed by data on $\ell A$ DIS and the proton-nucleus $(p A)$ Drell-Yan process-, the gluon content of the nuclei is even less known. To compensate this lack of constraints, both most recent global next-to-leading order (NLO) analyses of nPDFs, nCTEQ15 [18], and EPPS16 [17], used RHIC pion and LHC jet data (in case of EPPS16) to constrain the gluon densities down to $x \sim 10^{-3}$. However, there is no data at $x \lesssim 10^{-3}$. Hence, we do not know anything about the gluon at small $x$; the gluon nPDFs in this region are obtained by extrapolating nPDFs from larger $x$ region. As such, they essentially depend on the parametrizations of the $x$ dependence of nPDFs at the initial scale $\mu_{F, 0} \sim 1 \mathrm{GeV}$.

As discussed in Refs. [22,23], this lack of knowledge of the gluon nPDF is thus a priori not reflected by the set of error PDFs provided together with the best fit PDFs. Accordingly, increasing the flexibility of the initial nPDF parametrization leads to much larger uncertainties in this region as evidenced by the EPPS16 set as opposed to the EPS09 [24] and nCTEQ15 ones. Clearly, a determination of the small- $x$ gluon nPDFs and the reduction of their uncertainties is necessary for the heavy-ion phenomenology.

Recently, using heavy-flavor (HF) production at the LHC was proposed for an improved determination of the small- $x$ gluons in the proton [25-29]. We also noticed an earlier proposal [30]. Motivated by the results of these studies, we performed the first analysis of the impact of heavy-quark (onium) data in LHC proton-lead ( $p \mathrm{~Pb}$ ) collisions on the determination of nPDFs (nCTEQ15, EPPS16) as a way to constrain the small- $x$ gluon density in lead down to $x \simeq 7 \times 10^{-6}$.

The interpretation of our results depends on the reliability of nPDF factorization in the nuclear environment, which is a question of considerable theoretical and practical importance. In this context, we note that other cold-nuclear matter (CNM) effects [31-51] could become relevant in some specific conditions, in particular for the quarkonium case. In our study, they can be seen as higher-twist (HT) contributions and the use of leading-twist (LT) factorization becomes a working assumption to be tested. Once validated by data, as we will show, this assumption of LT factorization can be employed to learn about the internal structure of the nucleus.

Methodology-The cross sections measured in $p A$ collisions at colliders are nearly always normalized to the $p p$ ones $[7,52,53]$ since one is primarily interested in deviations from the free nucleon case, up to isospin effects. For DIS off a nucleus $A$, and thus $F_{2}^{\ell A}$, the NMF $R\left[F_{2}\right]$ is directly related to the modification $R_{q}^{A}$ of the (anti)quark nPDF compared to its PDF. For the gluons, one similarly defines $R_{g}^{A}$ entering theoretical evaluations of the NMF $R_{p A} \equiv d \sigma_{p A} /\left(A \times d \sigma_{p p}\right)$, which can be differential in the transverse momentum $\left(P_{T, \mathcal{H}}\right)$ or the center-of-momentum (c.m.s.) rapidity $y_{\text {c.m.s. }, \mathcal{H}}$ of the hadron $\mathcal{H}$. The nPDF sets provide parametrization of $R_{g}^{A}$ at any $x$ and scale. In the absence of nuclear effects, $R_{g}^{A}=1$ and we observe $R_{p A}\left(O_{\mathcal{H}}\right) \simeq 1$. Unlike the simple case of $F_{2}$ at leading order, $R_{g}^{A}$ enters $R_{p A}$ via a convolution which requires a control of the parton-scattering kinematics.

The focus on $R_{p A}$ has several advantages. It allows us to leave aside, in the theory evaluation, the proton PDF uncertainty at very small $x$ which may not always be negligible. Second, $R_{p A}$ is in general less sensitive to QCD corrections which may affect the normalization of the cross-section predictions. Third, some experimental uncertainties cancel in $R_{p A}$ and, at the LHC, $R_{p \mathrm{~Pb}}$ is usually more precise than the corresponding $p \mathrm{~Pb}$ cross sections.

To connect $R_{p A}$ and $R_{g}^{A}$, we will use the data-driven approach [54-56] where the parton-scattering-matrix elements squared $|A|^{2}$ are parametrized into empirical functions and determined from $p p$ data assuming a $2 \rightarrow 2$ kinematics together with given proton PDF, where we choose CT14NLO [1]. It was first motivated to bypass the complications inherent to our lack of understanding of the quarkoniumproduction mechanisms (see, e.g., Refs. [7,57]) whereas it suffices to evaluate the nPDF effects in $R_{p A}$. Such an approach also applies to open HF hadrons [54]. In the latter cases, full-fledged perturbative QCD computations exist [58-71] which we have used to validate the method. As in Ref. [54], we use a specific functional form for $|A|^{2}$ proposed in Ref. [72] to model single-quarkonium hadroproduction for double-parton scattering [72-76], which is sufficiently flexible to give a good description of single-inclusive-particle production.

There are several advantages in using this approach: (i) the uncertainty in the $p p$ cross section is controlled by the measured data, (ii) it can be applied to any singleinclusive-particle spectrum as long as the relative weights of the different channels (parton luminosities times $|A|^{2}$ ) are known, and (iii) the event generation is much faster than with QCD-based codes, allowing us to study several nPDFs with several scale choices in an acceptable amount of computing time. Indeed, to quantify the intrinsic theoretical uncertainty from the factorization scale $\mu_{F}$, we have varied it about a default scale $\mu_{0}$ as $\mu_{F}=\xi \mu_{0}$ with $\xi=0.5,1.0$, 2.0. $\mu_{0}^{2}$ was taken to be $M_{\mathcal{Q}}^{2}+P_{T, \mathcal{Q}}^{2}$ for $\mathcal{Q}=(J / \psi, \Upsilon)$, $4 M_{D}^{2}+P_{T, D}^{2}$ for $D^{0}$, and $4 M_{B}^{2}+\left(M_{B} / M_{J / \psi}\right)^{2} \times P_{T, J / \psi}^{2}$ for $B \rightarrow J / \psi$.

Compared to Ref. [54], the $p p$ baseline study was improved. For the first time, we considered the $B \rightarrow$ $J / \psi$ data. For $D^{0}, J / \psi$ and $\Upsilon(1 S)$, we advanced the scale study with a variation in the $p p$ baseline itself and not only in $R_{g}^{\mathrm{Pb}}\left(x, \mu_{F}\right)$, where $p p$ fits were done with each scale choice. As what concerns the $R_{p \mathrm{~Pb}}$ results, we checked that, for the cases of $D^{0}$ and $B \rightarrow J / \psi$ production, the scale uncertainty is nearly identical to that with the "fixed-orderplus-next-to-leading log" (FONLL) [64-66] calculation (see a comparison in the Supplemental Material [77]). 
As expected, FONLL gives much larger scale uncertainties on the yields.

As announced, to study the impact of HF experimental data on the gluon nPDF determination without performing a full fit, we employed the Bayesian-reweighting method [78-83]. This method is a direct application of Bayes theorem allowing one to include new data into a given PDF analysis without a fit. For the present study, we followed the same approach as in Ref. [83]. Since both nCTEQ15 and EPPS16 are Hessian nPDFs, we converted the Hessian error PDFs into $10^{4}$ Monte Carlo replicas, representing the underlying probability distribution [84]. For each PDF replica, one computes the $\chi^{2}$ of the considered data which is used to reweight them. Replicas describing better the data get larger weights than those unfavored by them. Hence, one obtains a modified probability distribution of the nPDFs like a fit would do.

Data selection-Like for all global PDF fits, a data selection is in order to avoid HT corrections. In our case, it is also important to select a kinematical region where gluon fusion dominates and other effects are negligible. As such, we considered the HF production in $p A$ collisions at LHC energies. In the quarkonium case, due to the large Lorentz boost at these energies, the heavy-quark pair remains almost pointlike all along its way through the nuclear matter. Therefore, breakup [85,86], thought to be important at lower energies, is negligible at the LHC. We focused on $J / \psi$ and $\Upsilon(1 S)$ to limit the contamination by possible comover effects [33-36,87], on the more fragile excited states [ $\psi(2 S), \Upsilon(2 S), \Upsilon(3 S)]$.

Overall, this gives the ALICE [88] and LHCb [89] $D^{0}$ data; the ALICE $[90,91]$ and LHCb $[92,93] J / \psi$ data; the LHCb [93] $B \rightarrow J / \psi$ data; the ALICE [94], ATLAS [95], and $\mathrm{LHCb}[96] \Upsilon(1 S)$ data. We could also add the $d \mathrm{Au}$ $J / \psi$ RHIC data. Instead, we preferred to focus on the LHC data at 5 and $8 \mathrm{TeV}$ and to use the RHIC [97,98] and the new LHC $[99,100]$ ones as cross checks.

Results-Figures (1a-1d) show a representative comparison of our theoretical calculations with the data for $D^{0}$, $J / \psi, B \rightarrow J / \psi$ and $\Upsilon(1 S)$. The NMF obtained with nCTEQ15 and EPPS16 have significantly different central values and uncertainties but both agree with the data. This observation is striking as the used gluon nPDFs were derived from totally different observables like DIS and Drell-Yan processes, and yet they allow us to reproduce the most important feature of the data [54] which makes our reweighting analysis meaningful. We see this as a confirmation of the LT factorization (see also Refs. [101-104]).

As for the reweighting results (gray-blue hatched bands in Figs. [1(a)-1(d)], if we could simply fix the scale to a single value for each particle, the LHC $R_{p \mathrm{~Pb}}$ data for prompt $D^{0}$ and $J / \psi$ would reduce the uncertainties of the gluon density by a factor 3 for EPPS16 and 2 for nCTEQ15 down to $x \simeq 7 \times 10^{-6}$ [compare the gray-blue and red hatched bands in Figs. 1(a) and 1(d)]. The current $B \rightarrow J / \psi$ and
$\Upsilon(1 S)$ data do not constrain the gluon nPDFs due to their large uncertainties and relatively large scales. Yet, the larger samples collected at $8 \mathrm{TeV}$ should improve the situation.

We now discuss the scale uncertainties and recall that $d \sigma_{p \mathrm{~Pb}} \sim f_{g}^{p}\left(f_{g}^{p} R_{g}^{\mathrm{Pb}}\right) \otimes|A|^{2}$. Because of QCD evolution, a larger $\mu_{F}$ implies a $R_{g}^{\mathrm{Pb}}$ closer to unity together with a smaller PDF uncertainty. Indeed, the bands in Figs. 1(a) -1 (d) are closer to unity and shrink from $\mu_{F}=$ $0.5 \mu_{0}$ to $\mu_{F}=2 \mu_{0}$. For nCTEQ15, such variations for $D^{0}$ and $J / \psi$ are even similar to the nPDF uncertainty itself.

Clearly, such a scale ambiguity should impact the reweighting results even though the (gray-blue) reweighted bands seems not to show such a sensitivity. It is perfectly normal since the replicas are to match the data. The key point is that they match it at different scales. Consequently, when the reweighted bands are evolved to a common scale $\mu_{F}=2 \mathrm{GeV}$, the reweighted nPDF uncertainties obtained with different scales do not superimpose (compare the black, blue, and green bands in Figs. 1(e)-1(f)).

The envelope of these scale-induced variations is about twice as large as their width for the $D^{0}$ and $J / \psi$ cases, confirming that the scale uncertainty must be accounted for to obtain reliable uncertainties from these precise data. For the heavier bottom(onium) states, the scale uncertainty is not only much smaller than the nPDF uncertainties but also very small in absolute value, which implies that more precise data could play a major role for a precision determination of the gluon nPDF at small $x$.

Despite these uncertainties, our results are striking: the $D^{0}$ and $J / \psi$ data point to the same magnitude of $R_{g}^{\mathrm{Pb}}$ and their inclusion in the EPPS16 fit would likely result in a considerable reduction of its gluon uncertainty by a factor as large as 1.7, see Fig. 1(f). For nCTEQ15, the effect seems less spectacular but we should recall that the original nCTEQ15 values at $x$ below $10^{-3}$ are pure extrapolations. The dashed red lines in Fig. 1(e) illustrates this by showing two equally good fits [22], which are now excluded by the LHC HF $p \mathrm{~Pb}$ data. Overall, the nCTEQ15 extrapolation to small $x$ is unexpectedly well confirmed by the charm(onium) data.

Beside the mere observations of the nPDF-uncertainty reduction, our results have two important physics interpretations. First, the LHC $p \mathrm{~Pb} \mathrm{HF}$ data give us the first real observation of gluon shadowing at small $x$ with $R_{g}^{\mathrm{Pb}}$ smaller than unity - the no-shadowing null-hypothesis-by more than 11.7 (10.9) and 7.3 (7.1) $\sigma$ at $x=10^{-5}$ and $\mu_{F}=$ $2 \mathrm{GeV}$ for nCTEQ15 and EPPS16 using $D^{0}(J / \psi)$ data [see Figs. 1(e)-1(f), left panels]. Our results thus quantitatively confirm the qualitative observations of [103-105] indirectly made from $J / \psi$ photoproduction on lead, which strictly speaking is sensitive to generalized parton distributions-not nPDFs-and suffers from significant scale uncertainties [106,107]. Second, our analysis corroborates the existence of a gluon antishadowing [108]: $R_{g}^{\mathrm{Pb}}>1$ for 


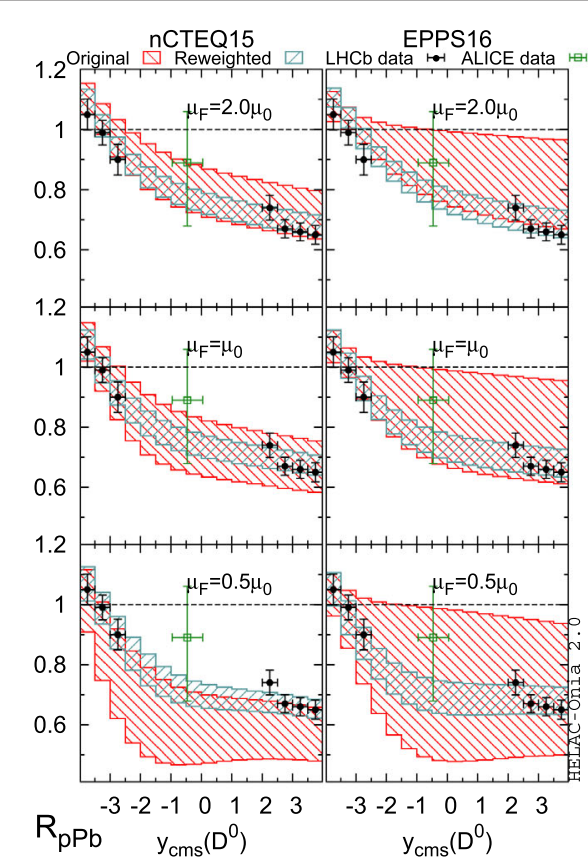

(a) Prompt $D^{0}$

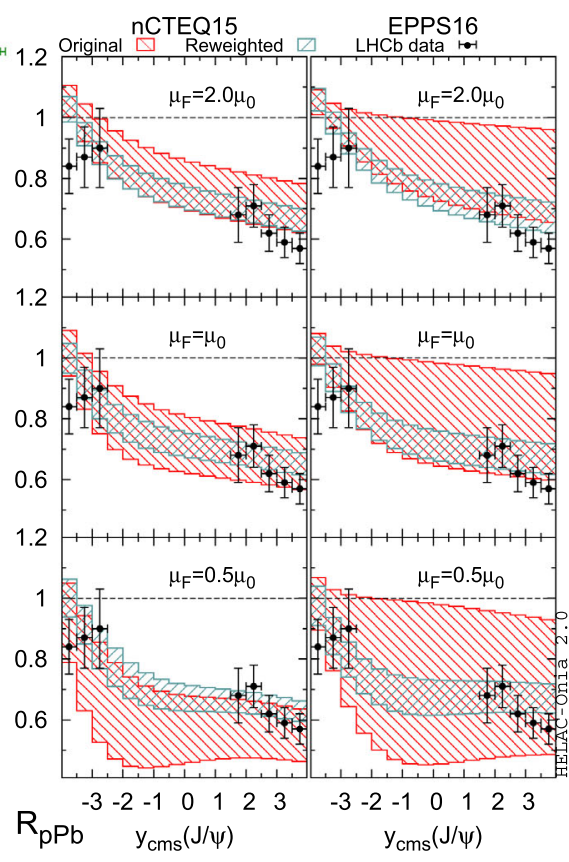

(b) Prompt $J / \psi$

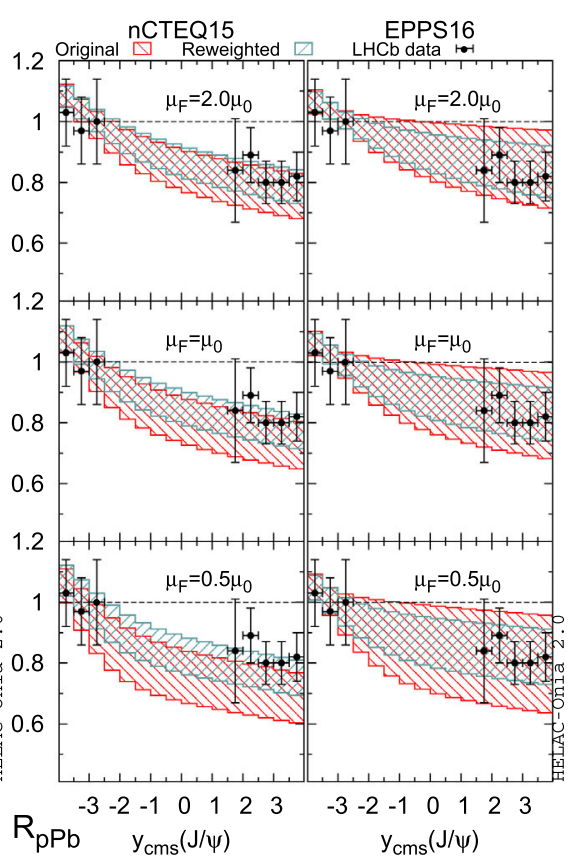

(c) $B \rightarrow J / \psi$

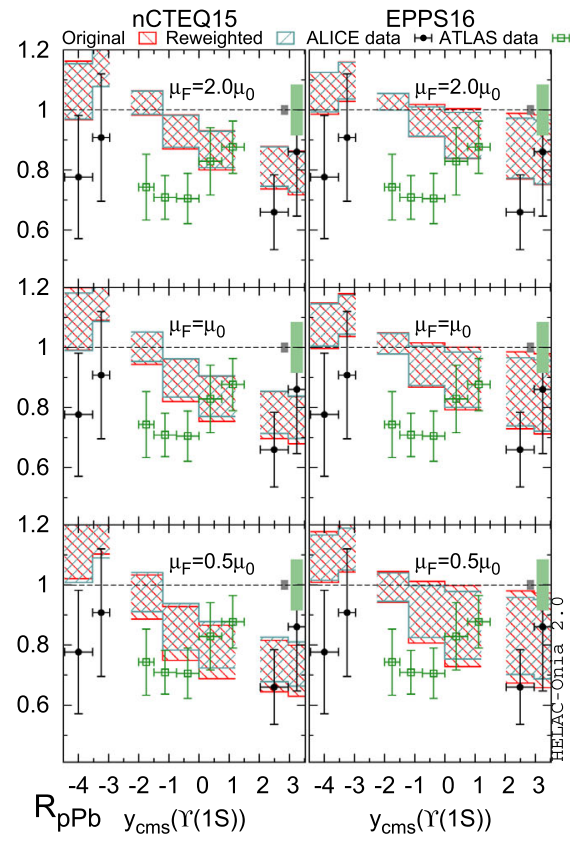

(d) $\Upsilon(1 S)$

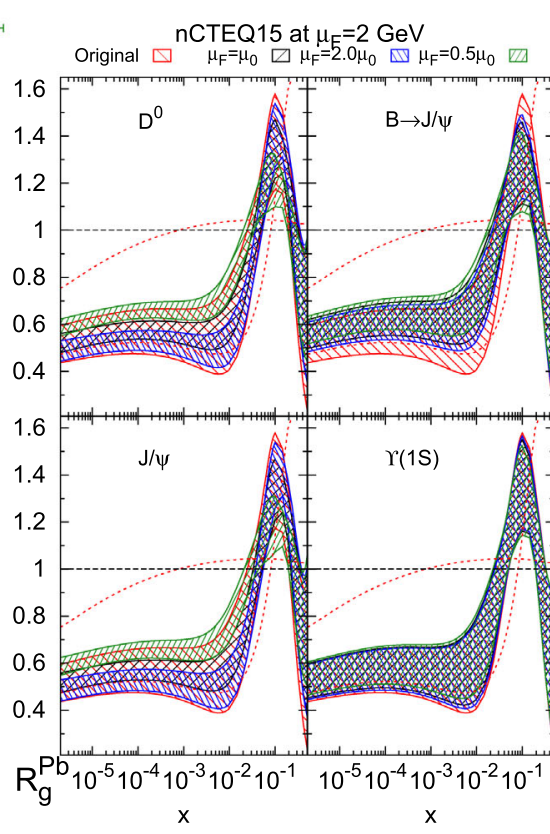

(e) nCTEQ15 nPDF

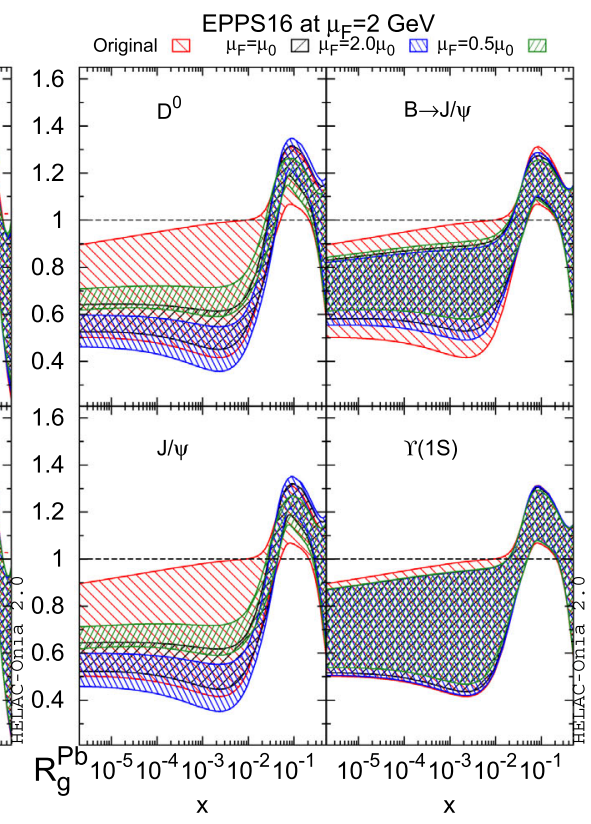

(f) EPPS16 nPDF

FIG. 1. Selected $R_{p \mathrm{~Pb}}$ results before and after reweighting for (a) prompt $D^{0}$, (b) prompt $J / \psi$, (c) $B \rightarrow J / \psi,(\mathrm{d}) \Upsilon(1 S)$ as well as the final reweighted nPDF uncertainties (e) nCTEQ15 and (f) EPPS16 with constraints from both $R_{p \text { Pb }}$ vs $P_{T, \mathcal{H}}$ and $y_{\text {c.m.s., } \mathcal{H}}$ data. The shown experimental data are from Refs. $[89,93,94,99,100]$. The error bands due to nPDF uncertainty are given at $68 \%$ C.L.

$x \simeq 0.1$. This can be seen in Figs. 1(e) and 1(f) where the error band after reweighting is smaller and more clearly separated from unity. The analyzed LHC heavy quark (onium) data cover the $x$ region $7 \times 10^{-6} \lesssim x \lesssim 0.1$. It is an interesting question how much of the antishadowing can be explained by direct data constraints in the region $x \lesssim 0.1$ and how much of the effect is indirectly driven by the momentum sum rule correlating a strong suppression at small $x$ with an enhancement in the antishadowing region. We leave this question open for a future publication.

Finally, we consider the global coherence of the HF constraints with other data (to be) included in nPDF global fits. We do it with nCTEQ15 of which 2 of us are authors. We thus have all the data at hand. First, let us observe that 
the agreement with the DIS NMC data [109], the only DIS set with a mild sensitivity to the gluon distribution, is not degraded in a statistically significant way. The original $\chi^{2} / N_{\text {data }}, 0.58$, becomes $(0.81,0.58,0.57)$ for $D^{0}$ with $\xi=(0.5,1,2)$, and is similar for other hadrons. Clearly, the inclusion of HF data does not create any tension with the DIS data. One can also make a similar comparison for the $W / Z p \mathrm{~Pb}$ LHC data whose impact on nCTEQ15 was recently studied [83]. The $\chi^{2} / N_{\text {data }}$ of these data was found to be 2.43 , and after our HF reweighting it becomes (2.14, $2.49,3.11)$ for $D^{0}$. With the same caveats as above, our reweighted nPDFs do not change the theory-data compatibility with the LHC $W / Z$ data. The $\chi^{2} / N_{\text {data }}$ of the $J / \psi$ PHENIX $R_{d \mathrm{Au}}$ results [97,98] with nCTEQ15 is (3.58, $2.55,3.12)$ and after our $J / \psi$ reweighting becomes $(1.81$, $2.38,2.77)$. This confirms the global coherence of the HF constraints. Tables of these $\chi^{2}$ values can be found as Supplemental Material [77].

Conclusion-In this Letter, we used, for the first time, experimental data for the inclusive $\operatorname{HF}\left[D^{0}, J / \psi, B \rightarrow J / \psi\right.$, $\Upsilon(1 S)]$ production in $p \mathrm{~Pb}$ collisions at the LHC to improve our knowledge of the gluon density inside heavy nuclei. We compared the data with computations obtained in the standard LT factorization framework endowed with the two most recent globally fit nPDFs (nCTEQ15, EPPS16). No other nuclear effects were included which are supposed to be of HT origin and hence suppressed as inverse powers of the hard scale. We found a good description of the LHC data with both nCTEQ15 and EPPS16 nPDFs validating our theoretical framework.

By performing a Bayesian-reweighting analysis and studying the scale uncertainties, we demonstrated that the existing heavy quark(onium) data can significantlyand coherently-reduce the uncertainty of the gluon density down to $x \simeq 7 \times 10^{-6}$. For charm(onium), the gluons are shadowed with a statistical significance beyond $7 \sigma$ at $\mu_{F}=2 \mathrm{GeV}$ and $x=10^{-5}$. These data should thus be included in the next generation of global nPDF analyses. While our results cannot rule out that other HT CNM effects were effectively "absorbed" into seemingly universal LT nPDFs, the observed consistent description of both the $D^{0}$ and $J / \psi$ data is far nontrivial since they may interact differently with the nuclear matter.

We are grateful to H. Paukkunen for the comments on using EPPS16 grids, to M. Cacciari for the private FONLL code, and to F. Arleo, E. G. Ferreiro, and P. Zurita for useful discussions. The work is supported by the ILP Labex (ANR-11-IDEX-0004-02, ANR-10-LABX-63) and the COPIN-IN2P3 Agreement.

[1] S. Dulat, T.-J. Hou, J. Gao, M. Guzzi, J. Huston, P. Nadolsky, J. Pumplin, C. Schmidt, D. Stump, and C. P. Yuan, New parton distribution functions from a global analysis of quantum chromodynamics, Phys. Rev. D 93, 033006 (2016).

[2] L. A. Harland-Lang, A. D. Martin, P. Motylinski, and R. S. Thorne, Parton distributions in the LHC era: MMHT 2014 PDFs, Eur. Phys. J. C 75, 204 (2015).

[3] R. D. Ball et al. (NNPDF Collaboration), Parton distributions from high-precision collider data, Eur. Phys. J. C 77, 663 (2017).

[4] A. Accardi, L. T. Brady, W. Melnitchouk, J. F. Owens, and N. Sato, Constraints on large- $x$ parton distributions from new weak boson production and deep-inelastic scattering data, Phys. Rev. D 93, 114017 (2016).

[5] S. Alekhin, J. Bluemlein, S. Moch, and R. Placakyte, Parton distribution functions, $\alpha_{s}$, and heavy-quark masses for LHC Run II, Phys. Rev. D 96, 014011 (2017).

[6] S. Alekhin et al., HERAFitter, Eur. Phys. J. C 75, 304 (2015).

[7] A. Andronic et al., Heavy-flavour and quarkonium production in the LHC era: from proton-proton to heavy-ion collisions, Eur. Phys. J. C 76, 107 (2016).

[8] J. J. Aubert et al. (European Muon Collaboration), The ratio of the nucleon structure functions $F 2_{n}$ for iron and deuterium, Phys. Lett. B 123, 275 (1983).

[9] M. S. Goodman et al., Observation of Shadowing in the Virtual Photon Total Hadronic Cross-Section on Nuclei, Phys. Rev. Lett. 47, 293 (1981).

[10] A. Bodek et al., A Comparison of the Deep Inelastic Structure Functions of Deuterium and Aluminum Nuclei, Phys. Rev. Lett. 51, 534 (1983).

[11] G. Bari et al. (BCDMS Collaboration), A measurement of nuclear effects in deep inelastic muon scattering on deuterium, nitrogen and iron targets, Phys. Lett. B 163, 282 (1985).

[12] A. C. Benvenuti et al. (BCDMS Collaboration), Nuclear effects in deep inelastic muon scattering on deuterium and iron targets, Phys. Lett. B 189, 483 (1987).

[13] J. Ashman et al. (European Muon Collaboration), Measurement of the ratios of deep inelastic muon-nucleus cross-sections on various nuclei compared to deuterium, Phys. Lett. B 202, 603 (1988).

[14] M. Arneodo et al. (European Muon Collaboration), Shadowing in deep inelastic muon scattering from nuclear targets, Phys. Lett. B 211, 493 (1988).

[15] D. F. Geesaman, K. Saito, and A. W. Thomas, The nuclear EMC effect, Annu. Rev. Nucl. Part. Sci. 45, 337 (1995).

[16] D. Higinbotham, G. A. Miller, O. Hen, and K. Rith, The EMC effect still puzzles after 30 years, CERN Cour. 53N4, 24 (2013).

[17] K. J. Eskola, P. Paakkinen, H. Paukkunen, and C. A. Salgado, EPPS16: Nuclear parton distributions with LHC data, Eur. Phys. J. C 77, 163 (2017).

[18] K. Kovarik et al., nCTEQ15-Global analysis of nuclear parton distributions with uncertainties in the CTEQ framework, Phys. Rev. D 93, 085037 (2016).

[19] H. Khanpour and S. Atashbar Tehrani, Global analysis of nuclear parton distribution functions and their uncertainties at next-to-next-to-leading order, Phys. Rev. D 93, 014026 (2016).

[20] D. de Florian, R. Sassot, P. Zurita, and M. Stratmann, Global analysis of nuclear parton distributions, Phys. Rev. D 85, 074028 (2012). 
[21] M. Hirai, S. Kumano, and T. H. Nagai, Determination of nuclear parton distribution functions and their uncertainties in next-to-leading order, Phys. Rev. C 76, 065207 (2007).

[22] T. Stavreva, I. Schienbein, F. Arleo, K. Kovarik, F. Olness, J. Y. Yu, and J. F. Owens, Probing gluon and heavy-quark nuclear PDFs with $\gamma+\mathrm{Q}$ production in $\rho A$ collisions, J. High Energy Phys. 01 (2011) 152.

[23] I. Helenius, H. Paukkunen, and N. Armesto, nPDF constraints from the large hadron electron collider, Proc. Sci., DIS2016 (2016) 276.

[24] K. J. Eskola, H. Paukkunen, and C. A. Salgado, EPS09: A new generation of NLO and LO nuclear parton distribution functions, J. High Energy Phys. 04 (2009) 065.

[25] O. Zenaiev et al. (PROSA Collaboration), Impact of heavy-flavour production cross sections measured by the $\mathrm{LHCb}$ experiment on parton distribution functions at low x, Eur. Phys. J. C 75, 396 (2015).

[26] R. Gauld, J. Rojo, L. Rottoli, and J. Talbert, Charm production in the forward region: constraints on the small-x gluon and backgrounds for neutrino astronomy, J. High Energy Phys. 11 (2015) 009.

[27] M. Cacciari, M. L. Mangano, and P. Nason, Gluon PDF constraints from the ratio of forward heavy-quark production at the LHC at $\sqrt{S}=7$ and $13 \mathrm{TeV}$, Eur. Phys. J. C 75, 610 (2015).

[28] R. Gauld and J. Rojo, Precision determination of the small$x$ gluon from charm production at LHCb, Phys. Rev. Lett. 118, 072001 (2017).

[29] E. G. de Oliveira, A. D. Martin, and M. G. Ryskin, Low $\mathrm{x}$ gluons determined by open charm production, arXiv: 1705.08845 .

[30] R. Gauld, Forward $D$ predictions for $p \mathrm{~Pb}$ collisions, and sensitivity to cold nuclear matter effects, Phys. Rev. D 93, 014001 (2016).

[31] C. Gerschel and J. Hufner, A contribution to the suppression of the $J \psi$ meson produced in high-energy nucleusnucleus collisions, Phys. Lett. B 207, 253 (1988).

[32] R. Vogt, $J / \psi$ production and suppression, Phys. Rep. 310, 197 (1999).

[33] E. G. Ferreiro, Excited charmonium suppression in proton-nucleus collisions as a consequence of comovers, Phys. Lett. B 749, 98 (2015).

[34] A. Capella and E. G. Ferreiro, $J / \psi$ suppression at $\sqrt{s}=$ $200-\mathrm{GeV}$ in the comovers interaction model, Eur. Phys. J. C 42, 419 (2005).

[35] A. Capella, E. G. Ferreiro, and A. B. Kaidalov, Nonsaturation of the $J / \psi$ Suppression at Large Transverse Energy in the Comovers Approach, Phys. Rev. Lett. 85, 2080 (2000).

[36] S. Gavin and R. Vogt, $J / \psi$ suppression from hadronnucleus to nucleus-nucleus collisions, Nucl. Phys. B345, 104 (1990).

[37] F. Arleo and S. Peigne, $J / \psi$ Suppression in $p$ - $A$ Collisions from Parton Energy Loss in Cold QCD Matter, Phys. Rev. Lett. 109, 122301 (2012).

[38] R. Sharma and I. Vitev, High transverse momentum quarkonium production and dissociation in heavy ion collisions, Phys. Rev. C 87, 044905 (2013).

[39] F. Arleo, S. Peigne, and T. Sami, Revisiting scaling properties of medium-induced gluon radiation, Phys. Rev. D 83, 114036 (2011).
[40] S. J. Brodsky and P. Hoyer, A Bound on the energy loss of partons in nuclei, Phys. Lett. B 298, 165 (1993).

[41] S. Gavin and J. Milana, Energy loss at large $x_{F}$ in nuclear collisions, Phys. Rev. Lett. 68, 1834 (1992).

[42] S. J. Brodsky and P. Hoyer, The Nucleus as a Color Filter in QCD Decays: Hadroproduction in Nuclei, Phys. Rev. Lett. 63, 1566 (1989).

[43] B. Ducloué, T. Lappi, and H. Mäntysaari, Forward $J / \psi$ production in proton-nucleus collisions at high energy, Phys. Rev. D 91, 114005 (2015).

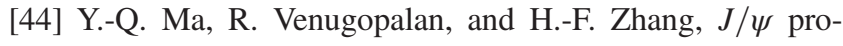
duction and suppression in high energy proton-nucleus collisions, Phys. Rev. D 92, 071901 (2015).

[45] H. Fujii and K. Watanabe, Heavy quark pair production in high energy $\rho A$ collisions: Quarkonium, Nucl. Phys. A915, 1 (2013).

[46] J.-W. Qiu, P. Sun, B.-W. Xiao, and F. Yuan, Universal suppression of heavy quarkonium production in $\rho A$ collisions at low transverse momentum, Phys. Rev. D 89, 034007 (2014).

[47] B. Kopeliovich, A. Tarasov, and J. Hufner, Coherence phenomena in charmonium production off nuclei at the energies of RHIC and LHC, Nucl. Phys. A696, 669 (2001).

[48] E. G. Ferreiro, F. Fleuret, J. P. Lansberg, and A. Rakotozafindrabe, Cold nuclear matter effects on $J / \psi$ production: Intrinsic and extrinsic transverse momentum effects, Phys. Lett. B 680, 50 (2009).

[49] E. G. Ferreiro, F. Fleuret, J. P. Lansberg, N. Matagne, and A. Rakotozafindrabe, $\Upsilon$ production in $p(d) A$ collisions at RHIC and the LHC, Eur. Phys. J. C 73, 2427 (2013).

[50] E. G. Ferreiro, F. Fleuret, J. P. Lansberg, and A. Rakotozafindrabe, Impact of the nuclear modification of the gluon densities on $J / \psi$ production in $p \mathrm{~Pb}$ collisions at $\sqrt{s_{N N}}=5$ TeV, Phys. Rev. C 88, 047901 (2013).

[51] R. Vogt, Cold nuclear matter effects on $J / \psi$ and $\Upsilon$ production at the LHC, Phys. Rev. C 81, 044903 (2010).

[52] J. L. Albacete et al., Predictions for $p+\mathrm{Pb}$ collisions at $\sqrt{s_{N N}}=5 \mathrm{TeV}$ : Comparison with data, Int. J. Mod. Phys. E 25, 1630005 (2016).

[53] J. L. Albacete et al., Predictions for cold nuclear matter effects in $p+\mathrm{Pb}$ collisions at $\sqrt{s_{N N}}=8.16 \mathrm{TeV}$, Nucl. Phys. A972, 18 (2018).

[54] J.-P. Lansberg and H.-S. Shao, Towards an automated tool to evaluate the impact of the nuclear modification of the gluon density on quarkonium, D and B meson production in proton-nucleus collisions, Eur. Phys. J. C 77, 1 (2017).

[55] H.-S. Shao, HELAC-Onia: An automatic matrix element generator for heavy quarkonium physics, Comput. Phys. Commun. 184, 2562 (2013).

[56] H.-S. Shao, HELAC-Onia 2.0: An upgraded matrixelement and event generator for heavy quarkonium physics, Comput. Phys. Commun. 198, 238 (2016).

[57] N. Brambilla et al., Heavy quarkonium: Progress, puzzles, and opportunities, Eur. Phys. J. C 71, 1534 (2011).

[58] B. A. Kniehl, G. Kramer, I. Schienbein, and H. Spiesberger, Inclusive $\mathrm{D}^{* \pm}$ production in $p \bar{p}$ collisions with massive charm quarks, Phys. Rev. D 71, 014018 (2005).

[59] B. A. Kniehl, G. Kramer, I. Schienbein, and H. Spiesberger, Collinear subtractions in hadroproduction of heavy quarks, Eur. Phys. J. C 41, 199 (2005). 
[60] B. A. Kniehl, G. Kramer, I. Schienbein, and H. Spiesberger, Inclusive charmed-meson production at the CERN LHC, Eur. Phys. J. C 72, 2082 (2012).

[61] B. A. Kniehl, G. Kramer, I. Schienbein, and H. Spiesberger, Inclusive B-meson production at the LHC in the GM-VFN scheme, Phys. Rev. D 84, 094026 (2011).

[62] B. A. Kniehl, G. Kramer, I. Schienbein, and H. Spiesberger, Inclusive $B$-meson production at small $p_{T}$ in the generalmass variable-flavor-number scheme, Eur. Phys. J. C 75, 140 (2015).

[63] J. Alwall, R. Frederix, S. Frixione, V. Hirschi, F. Maltoni, O. Mattelaer, H. S. Shao, T. Stelzer, P. Torrielli, and M. Zaro, The automated computation of tree-level and nextto-leading order differential cross sections, and their matching to parton shower simulations, J. High Energy Phys. 07 (2014) 079.

[64] M. Cacciari, M. Greco, and P. Nason, The $p_{T}$ spectrum in heavy flavor hadroproduction, J. High Energy Phys. 05 (1998) 007.

[65] M. Cacciari, S. Frixione, and P. Nason, The $p_{T}$ spectrum in heavy flavor photoproduction, J. High Energy Phys. 03 (2001) 006.

[66] M. Cacciari, S. Frixione, N. Houdeau, M. L. Mangano, P. Nason, and G. Ridolfi, Theoretical predictions for charm and bottom production at the LHC, J. High Energy Phys. 10 (2012) 137.

[67] M. L. Mangano, P. Nason, and G. Ridolfi, Heavy quark correlations in hadron collisions at next-to-leading order, Nucl. Phys. B373, 295 (1992).

[68] P. Nason, A New method for combining NLO QCD with shower Monte Carlo algorithms, J. High Energy Phys. 11 (2004) 040.

[69] S. Frixione, P. Nason, and C. Oleari, Matching NLO QCD computations with Parton Shower simulations: the POWHEG method, J. High Energy Phys. 11 (2007) 070.

[70] S. Frixione and B. R. Webber, Matching NLO QCD computations and parton shower simulations, J. High Energy Phys. 06 (2002) 029.

[71] S. Frixione, P. Nason, and B. R. Webber, Matching NLO QCD and parton showers in heavy flavor production, J. High Energy Phys. 08 (2003) 007.

[72] C. H. Kom, A. Kulesza, and W. J. Stirling, Pair production of $J / \psi$ as a probe of double parton scattering at $\mathrm{LHCb}$, Phys. Rev. Lett. 107, 082002 (2011).

[73] J.-P. Lansberg and H.-S. Shao, J/ $\psi$-pair production at large momenta: Indications for double parton scatterings and large $\alpha_{s}^{5}$ contributions, Phys. Lett. B 751, 479 (2015).

[74] J.-P. Lansberg and H.-S. Shao, Double-quarkonium production at a fixed-target experiment at the LHC (AFTER@LHC), Nucl. Phys. B900, 273 (2015).

[75] H.-S. Shao and Y.-J. Zhang, Complete study of hadroproduction of a $\Upsilon$ meson associated with a prompt $J / \psi$, Phys. Rev. Lett. 117, 062001 (2016).

[76] C. Borschensky and A. Kulesza, Double parton scattering in pair production of $J / \psi$ mesons at the LHC revisited, Phys. Rev. D 95, 034029 (2017).

[77] See Supplemental Material at http://link.aps.org/ supplemental/10.1103/PhysRevLett.121.052004 for the validation of our method with FONLL and for more details on our study including the corresponding $\chi^{2}$ values, the effective numbers of replicas in each considered case and the fit values of the parameters appearing in our datadriven approach.

[78] W. T. Giele and S. Keller, Implications of hadron collider observables on parton distribution function uncertainties, Phys. Rev. D 58, 094023 (1998).

[79] R. D. Ball, V. Bertone, F. Cerutti, L. Del Debbio, S. Forte, A. Guffanti, J. I. Latorre, J. Rojo, and M. Ubiali (NNPDF Collaboration), Reweighting NNPDFs: The W lepton asymmetry, Nucl. Phys. B849, 112 (2011); Erratum, Nucl. Phys.B855, 927 (2012)].

[80] R. D. Ball, V. Bertone, F. Cerutti, L. Del Debbio, S. Forte, A. Guffanti, N. P. Hartland, J. I. Latorre, J. Rojo, and M. Ubiali, Reweighting and unweighting of parton distributions and the LHC W lepton asymmetry data, Nucl. Phys. B855, 608 (2012).

[81] N. Sato, J. F. Owens, and H. Prosper, Bayesian reweighting for global fits, Phys. Rev. D 89, 114020 (2014).

[82] H. Paukkunen and P. Zurita, PDF reweighting in the Hessian matrix approach, J. High Energy Phys. 12 (2014) 100.

[83] A. Kusina, F. Lyonnet, D. B. Clark, E. Godat, T. Jezo, K. Kovarik, F. I. Olness, I. Schienbein, and J. Y. Yu, Vector boson production in $p \mathrm{~Pb}$ and $\mathrm{PbPb}$ collisions at the LHC and its impact on nCTEQ15 PDFs, Eur. Phys. J. C 77, 488 (2017).

[84] The 32 (40) eigensets of these Hessian nPDFs are meant to display a $90 \%$ confidence level (C.L.) uncertainty with a tolerance $\Delta \chi^{2}=35$ (nCTEQ15) and 52 (EPPS16), respectively. Let us note that in what follows our results will be displayed at $68 \% \mathrm{CL}$, i.e., $1-\sigma$ which is more suitable for theory-data comparison with 1- $\sigma$ experimental uncertainties. This simply amounts to reduce the uncertainties by $\sqrt{2} \operatorname{erf}^{-1}(0.90) \simeq 1.645$.

[85] R. Vogt, Shadowing and absorption effects on $P / \psi$ production in dA collisions, Phys. Rev. C 71, 054902 (2005).

[86] C. Lourenco, R. Vogt, and H. K. Woehri, Energy dependence of $P / \psi$ absorption in proton-nucleus collisions, J. High Energy Phys. 02 (2009) 014.

[87] E. G. Ferreiro and J.-P. Lansberg, Is bottomonium suppression in proton-nucleus and nucleus-nucleus collisions at LHC energies due to the same effects?, arXiv:1804.04474.

[88] B. B. Abelev et al. (ALICE Collaboration), Measurement of prompt $D$-meson production in $p-P b$ collisions at $\sqrt{s_{N N}}=5.02 \mathrm{TeV}$, Phys. Rev. Lett. 113, 232301 (2014).

[89] R. Aaij et al. (LHCb Collaboration), Study of prompt $\mathrm{D}^{0}$ meson production in $p \mathrm{~Pb}$ collisions at $\sqrt{s_{\mathrm{NN}}}=5 \mathrm{TeV}$, J. High Energy Phys. 10 (2017) 090.

[90] J. Adam et al. (ALICE Collaboration), Rapidity and transverse-momentum dependence of the inclusive $J / \psi$ nuclear modification factor in $p \mathrm{~Pb}$ collisions at $\sqrt{s_{N N}}=5.02 \mathrm{TeV}$, J. High Energy Phys. 06 (2015) 055.

[91] B. B. Abelev et al. (ALICE Collaboration), $J / \psi$ production and nuclear effects in $p-P b$ collisions at $\sqrt{S_{N N}}=5.02 \mathrm{TeV}$, J. High Energy Phys. 02 (2014) 073.

[92] R. Aaij et al. (LHCb Collaboration), Study of $J / \psi$ production and cold nuclear matter effects in $p P b$ collisions at $\sqrt{s_{N N}}=5 \mathrm{TeV}$, J. High Energy Phys. 02 (2014) 072. 
[93] R. Aaij et al. (LHCb Collaboration), Prompt and nonprompt $J / \psi$ production and nuclear modification in $p \mathrm{~Pb}$ collisions at $\sqrt{s_{\mathrm{NN}}}=8.16 \mathrm{TeV}$, Phys. Lett. B 774, 159 (2017).

[94] B. B. Abelev et al. (ALICE Collaboration), Production of inclusive $\Upsilon(1 S)$ and $\Upsilon(2 S)$ in $p-P b$ collisions at $\sqrt{s_{\mathrm{NN}}}=\mathbf{5 . 0 2} \mathrm{TeV}$, Phys. Lett. B 740, 105 (2015).

[95] ATLAS Collaboration, Measurement of $\Upsilon(\mathrm{nS})$ production with $p+\mathrm{Pb}$ collisions at $\sqrt{s_{\mathrm{NN}}}=5.02 \mathrm{TeV}$ and $p p$ collisions at $\sqrt{s}=2.76 \mathrm{TeV}$, Report no. ATLASCONF-2015-050.

[96] R. Aaij et al. (LHCb Collaboration), Study of $\Upsilon$ production and cold nuclear matter effects in $p \mathrm{~Pb}$ collisions at $\sqrt{s_{N N}}=5 \mathrm{TeV}$, J. High Energy Phys. 07 (2014) 094.

[97] A. Adare et al. (PHENIX Collaboration), Cold Nuclear Matter Effects on $J / \psi$ Yields as a Function of Rapidity and Nuclear Geometry in Deuteron-Gold Collisions at $\sqrt{s_{N N}}=200 \mathrm{GeV}$, Phys. Rev. Lett. 107, 142301 (2011).

[98] A. Adare et al. (PHENIX Collaboration), Transversemomentum dependence of the $J / \psi$ nuclear modification in $d+\mathrm{Au}$ collisions at $\sqrt{s_{N N}}=200 \mathrm{GeV}$, Phys. Rev. C 87, 034904 (2013).

[99] M. Aaboud et al. (ATLAS Collaboration), Measurement of quarkonium production in proton-lead and proton-proton collisions at 5.02 TeV with the ATLAS detector, Eur. Phys. J. C78, 171 (2018).

[100] J. Adam et al. (ALICE Collaboration), D-meson production in $p$ - $\mathrm{Pb}$ collisions at $\sqrt{s_{\mathrm{NN}}}=5.02 \mathrm{TeV}$ and in pp collisions at $\sqrt{s}=7 \mathrm{TeV}$, Phys. Rev. C 94, 054908 (2016).
[101] L. Frankfurt, V. Guzey, and M. Strikman, Leading twist nuclear shadowing phenomena in hard processes with nuclei, Phys. Rep. 512, 255 (2012).

[102] L. Frankfurt, V. Guzey, and M. Strikman, Dynamical model of antishadowing of the nuclear gluon distribution, Phys. Rev. C 95, 055208 (2017).

[103] V. Guzey, E. Kryshen, M. Strikman, and M. Zhalov, Evidence for nuclear gluon shadowing from the ALICE measurements of $\mathrm{PbPb}$ ultraperipheral exclusive $\mathrm{J} / \psi$ production, Phys. Lett. B 726, 290 (2013).

[104] B. Abelev et al. (ALICE Collaboration), Coherent $J / \psi$ photoproduction in ultra-peripheral $\mathrm{Pb}-\mathrm{Pb}$ collisions at $\sqrt{s_{N N}}=2.76 \mathrm{TeV}$, Phys. Lett. B 718, 1273 (2013).

[105] V. Khachatryan et al. (CMS Collaboration), Coherent $J / \psi$ photoproduction in ultra-peripheral $\mathrm{PbPb}$ collisions at $\sqrt{s_{N N}}=2.76 \mathrm{TeV}$ with the CMS experiment, Phys. Lett. B 772, 489 (2017).

[106] D. Yu. Ivanov, A. Schafer, L. Szymanowski, and G. Krasnikov, Exclusive photoproduction of a heavy vector meson in QCD, Eur. Phys. J. C 34, 297 (2004); Erratum, Eur. Phys. J. C 75, 75E (2015).

[107] S. P. Jones, A. D. Martin, M. G. Ryskin, and T. Teubner, Exclusive $J / \psi$ and $\Upsilon$ photoproduction and the low $x$ gluon, J. Phys. G 43, 035002 (2016).

[108] L. L. Frankfurt, M. I. Strikman, and S. Liuti, Evidence for enhancement of gluon and valence quark distributions in nuclei from hard lepton nucleus processes, Phys. Rev. Lett. 65, 1725 (1990).

[109] M. Arneodo et al. (New Muon Collaboration), The $Q^{2}$ dependence of the structure function ratio $F_{2}{ }^{S n} / F_{2}{ }^{c}$ and the difference $R^{S n}-R^{c}$ in deep inelastic muon scattering, Nucl. Phys. B481, 23 (1996). 